\title{
Madera plástica con PET de post consumo y paja de trigo \\ Ángel García-Velázquez ${ }^{1}$, María Guadalupe Amado-Moreno', Marco \\ Antonio Casados-Pérez ${ }^{1}$ y Reyna Arcelia Brito-Páez ${ }^{1}$
}

\section{Resumen}

El objetivo de la investigación fue desarrollar un material compuesto de polietileno tereftalato (PET) de post consumo y paja de trigo, para la elaboración de madera plástica. El PET es el plástico de mayor volumen y tonelaje en los rellenos sanitarios y basureros de Mexicali, Baja California, México. Por otro lado, la paja de trigo es un desecho agrícola en el Valle de Mexicali. Lo anterior motivó la realización de la investigación, pues existen en la zona bajos porcentajes de reciclado del PET y paja de trigo para materiales compuestos. Se aprovechan principalmente las propiedades mecánicas del PET reforzado con paja de trigo pulverizada para elaborar el material compuesto. La madera plástica obtenida de estos dos materiales de desecho no presenta poros, se puede: taladrar, lijar, pulir, cortar con sierra manual o eléctrica, por lo que, puede utilizarse para la elaboración de tarimas industriales, mesas, sillas, libreros, escritorios, entre otras aplicaciones.

Palabras Clave: Material compuesto, PET de post consumo, madera plástica. 


\section{Abstract}

The aim of the research was to develop a composite of post-consumer polyethylene terephthalate (PET) and wheat Straw to produce wood plastic. PET is the plastic with more volume and tonnage in landfills and dumps of Mexicali, Baja California, Mexico. On the other hand, wheat straw is an agricultural waste from the Mexicali Valley. This led to conduct a research in the area since it shows low percentages of PET and wheat straw recycling for composites. The mechanical properties of PET reinforced with pulverized wheat straw are mainly used to prepare composites. Wood plastic obtained from these two waste materials has no pores and can be drilled, sanded, polished, cut with hand or power saw. Wood plastic can be used for producing industrial flooring, tables, chairs, bookcases, desks among others.

Keywords: composite, post-consumer PET, wood plastic. 


\section{Introducción}

Los primeros pobladores de lo que ahora es la ciudad de Mexicali, Baja California (B. C), México, se establecieron a la orilla del Río Nuevo. En el margen de este río crecía y sigue creciendo una planta endémica llamada "cachanilla", que al combinarse con lodo, sirvió como material para fabricar sus casas, siendo totalmente ecológicas. El aprovechamiento de los recursos disponibles ha estado presente entre los habitantes de la región.

Actualmente los pobladores de Mexicali, B. C., enfrentan problemas de contaminación derivados del consumo de productos embotellados y de la quema de residuos agrícolas.

El polietileno tereftalato, mejor conocido como PET, es uno de los materiales más utilizados en la industria embotelladora. Por la resistencia y liviandad del material, favorecen el manejo y distribución de productos, lo que ha impulsado el desarrollo de la industria al diversificar su producción. Sin embargo, derivado del alto nivel de consumo de productos embotellados, se tienen también, grandes cantidades de residuos de PET (García, 2006).

Los datos indican que los mexicanos consumen un promedio de $6.5 \mathrm{~kg} /$ año de PET, generando 103,000 toneladas de desechos del mismo. Los envases de PET ocupan entre el $2-4 \%$ en peso y entre el $7-10 \%$ en volumen en los rellenos sanitarios en el país. De estos sólo se recicla el 5.2 \% (Cox, 1952), (Flores, 2010).

Las botellas de PET se encuentran frecuentemente en los basureros públicos y rellenos sanitarios, contaminando ríos y mares (García C. , 2010).

La ciudad de Mexicali, B. C., al igual que otras ciudades del país, presenta contaminación por botellas de PET. Es por ello necesario su rehúso y/o reprocesado.

Otro problema que se presenta en esta ciudad es la contaminación del aire por la quema de residuos agrícolas, en particular la paja de trigo.

El volumen de contaminantes producidos en Mexicali, por la quema de paja de trigo presenta un factor de carga de 4.75 toneladas de desperdicio del cultivo por hectárea. En el Valle de Mexicali se siembran aproximadamente 273,643 hectáreas de trigo, muchas más que otros cultivos. Se calcula que se emiten a la atmósfera, 233, 19.5, y 8.75 kilogramos de contaminantes de CO, hidrocarburos $\mathrm{CnH} 2 \mathrm{n}+2$ y NO2 por hectárea quemada (Quintero, 2008).

El problema de contaminación en la ciudad exige la realización de investigaciones que favorezcan el rehúso y/o reprocesado de materiales de desecho. Es por ello que se realizó esta investigación, con la finalidad de aprovechar los materiales de desecho que se encuentran en la región, tanto el PET como el residuo de paja; siendo su reprocesamiento un área de oportunidad.

La investigación se llevó a cabo en una Institución de Educación Superior en México: el Instituto Tecnológico de Mexicali, en Baja California. En ella 
participaron académicos de la institución y estudiantes de ingeniería química.

El objetivo de la investigación fue desarrollar una placa de material compuesto con PET de post consumo y paja de trigo para obtener con su reprocesado una madera plástica.

El rehúso de las botellas de plástico y paja de trigo permitió elaborar un material compuesto que es aquel que se obtiene al mezclar dos o más materiales distintos sin que ocurra reacción química entre ellos. La combinación adecuada de ellos origina materiales con mejores propiedades que las partes que lo componen por separado. El material compuesto está constituido por el material de refuerzo y una matriz. El primero de ellos es el elemento que otorga la resistencia y el segundo actúa como aglutinante (Plueddemann, 1974), (Cox, 1952).

El material de refuerzo tiene como objeto soportar el esfuerzo aplicado, mientras que a la matriz le corresponde trasmitir de forma efectiva y uniforme el esfuerzo hacia todo el material (Sato, 1962), (Chon, 1980).

\section{Metodología}

El procedimiento general consistió en 5 etapas:

- Acopio, separación, lavado y molido de las botellas de PET.

- Acopio, molido y cernido de la paja de trigo.

- Extrusor utilizado para el mezclado del PET con paja de trigo.

- Diseño de experimentos para elaborar el material compuesto.

- Elaboración de probetas para realizar las pruebas de resistencia al impacto y dureza del material compuesto.

\section{Descripción de las etapas de la investigación.}

Etapa 1: Acopio, separación, lavado y molido de las botellas de PET.

La primera parte de esta etapa se inició con una campaña para acopio y recolección de botellas de todo tipo de plástico. Se colocaron contenedores en la Institución y se recopiló en parques, con familiares y en la comunidad. Se realizó un acopio de aproximadamente $560 \mathrm{~kg}$ de plástico en botella. En la Figura 1 se observa parte del plástico recolectado.

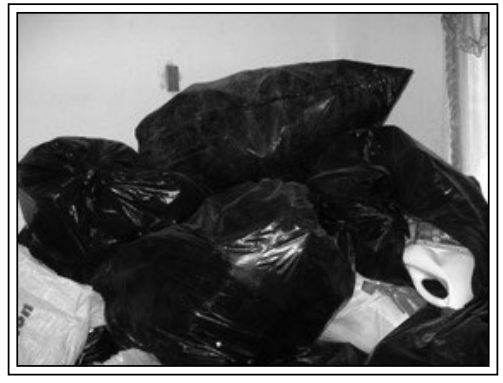

Figura 1.

Acopio de plástico en botella. 
Posteriormente se realizó la separación y lavado de las botellas de plástico, manualmente se separó las botellas de PET por colores. Se les retiró la etiqueta, tapones y la corona ya que estas están hechas de polietileno de alta densidad.

El lavado se realizó con agua corriente con el fin de eliminar impurezas, residuos de líquidos y todo aquel material incompatible con el PET que pudiera contaminar el proceso.

Las botellas limpias y por colores se separaron en bolsas para posteriormente molerse. Se trabajó con las botellas de PET transparentes para eliminar el factor de pigmentación en la fórmula del producto a obtener.

Se molió el plástico trasparente y limpio a un tamaño de partícula de 3 a $9.5 \mathrm{~mm}$ con el fin de facilitar su fundición en el extrusor y se almacenó en contenedores para su posterior procesado. En la Figura 2 se muestra el plástico limpio, el molino utilizado y el PET molido.

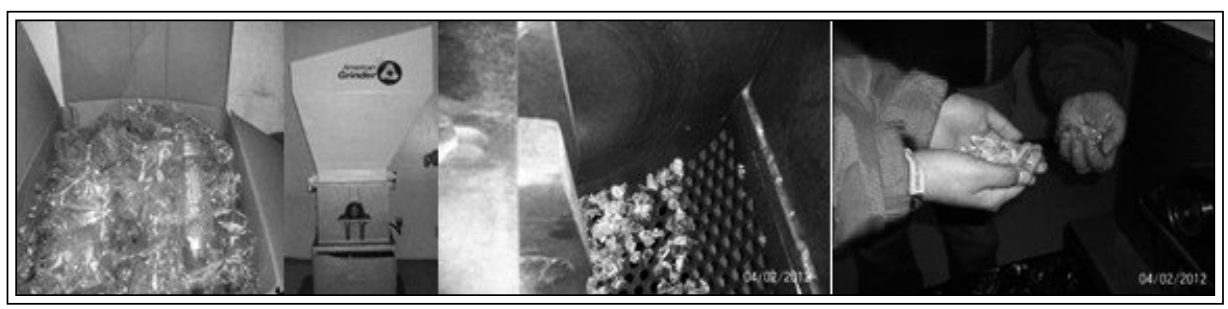

Figura 2. Molido del PET.

Etapa 2: Acopio, molido y cernido de la paja de trigo.

Se realizó el acopio de $200 \mathrm{~kg}$ de paja de trigo en pacas de $25 \mathrm{~kg}$, la cual se molió aplicando esfuerzos cortantes para deshacer por completo la paja, esto se realizó en un molino para semilla marca Swissmex Modelo LX 300 accionado por tractor, mostrado en la Figura 3.

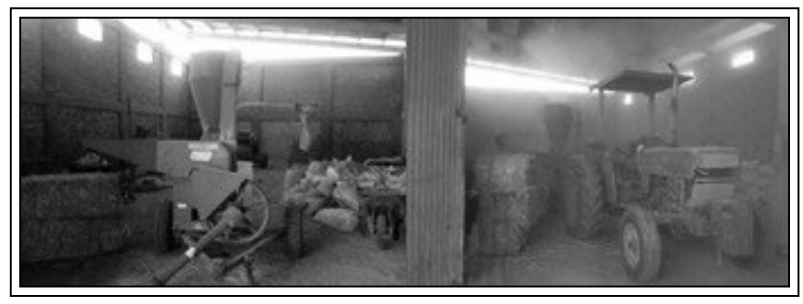

Figura 3. Molido de la paja de trigo.

La paja triturada y molida conservó una geometría alargada, por este motivo fue necesario cernir para separar partículas y facilitar su incorporación con el PET en la mezcla a fundir. La paja fue cernida en un tamizador para obtener tamaños de partícula con malla $14(1.41 \mathrm{~mm}), 20(0.84 \mathrm{~mm}), 30(0.59 \mathrm{~mm})$ y $40(0.42 \mathrm{~mm})$ como se observa en la Figura 4. 


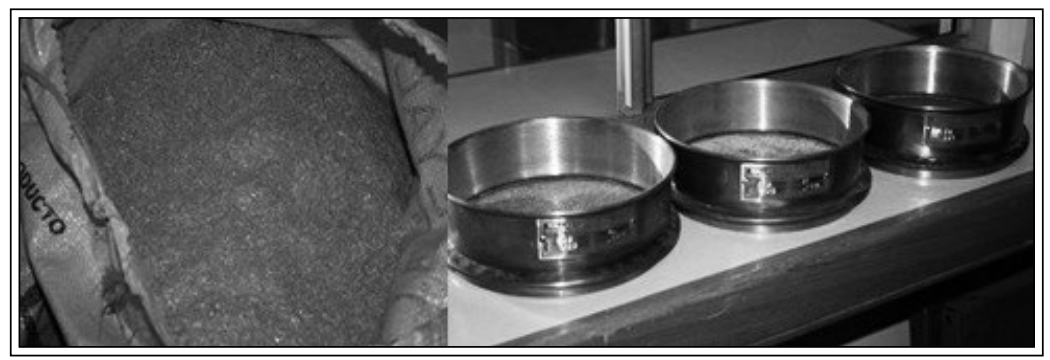

Figura 4. Paja cernida con malla 14, 20, 30 y 40.

Etapa 3: Extrusor utilizado para el mezclado del PET con paja de trigo.

Se preparó el extrusor y el sistema hidráulico mostrado en la figura 5, el extrusor cuenta con un tornillo con una configuración (longitud/diámetro) $\mathrm{L} / \mathrm{D}=20 \mathrm{sin}$ secciones de venteo en el cual se realizó la fundición y la mezcla de PET - paja de trigo. Se determinaron las temperaturas de calentamiento al cañón para obtener buena fluidez de la mezcla.

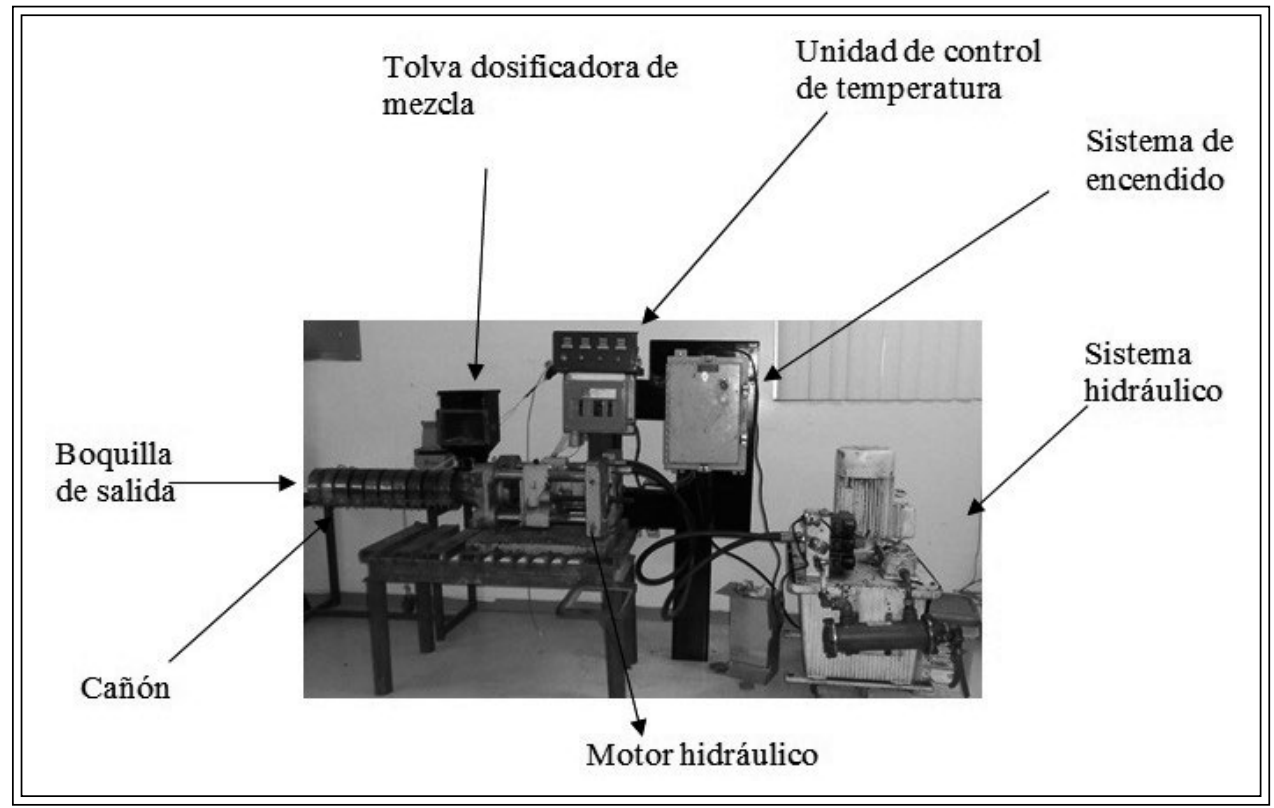

Figura 5. Extrusor-sistema hidráulico.

Etapa 4: Diseño de experimentos para elaborar el material compuesto de PET de post consumo y paja de trigo.

El diseño de experimentos se realizó basado en:

a) Porcentaje en peso de paja de trigo.

b) Tamaño de partícula de paja de trigo. 
En la Tabla 1 se muestra el diseño de experimentos, realizado con dos factores que fueron tamaño de partícula y porcentaje en peso de paja de trigo con cuatro niveles cada uno.

Tabla 1. Diseño de experimentos con dos factores (paja de trigo y tamaño de partícula).

\begin{tabular}{|c|c|c|c|c|}
\hline \multirow{2}{*}{$\begin{array}{l}\% \text { en peso } \\
\text { de Paja de trigo }\end{array}$} & \multicolumn{4}{|c|}{ Tamaño de Malla } \\
\hline & $\begin{array}{l}14 \\
(1.41 \mathrm{~mm})\end{array}$ & $\begin{array}{l}20 \\
(0.84 \mathrm{~mm})\end{array}$ & $\begin{array}{l}30 \\
(0.59 \mathrm{~mm})\end{array}$ & $\begin{array}{l}40 \\
(0.42 \mathrm{~mm})\end{array}$ \\
\hline \multicolumn{5}{|l|}{30} \\
\hline \multicolumn{5}{|l|}{40} \\
\hline \multicolumn{5}{|l|}{50} \\
\hline 60 & & & & \\
\hline
\end{tabular}

En esta investigación se utilizó la paja de trigo como material de refuerzo y el PET como material aglutinante o de unión. Se planteó no utilizar aditivos que encarecieran el proceso.

La mezcla de los componentes se realizó en un extrusor mono-husillo con relación $\mathrm{L} / \mathrm{D}=20$. Las temperaturas utilizadas en las tres zonas de calentamiento del extrusor fueron de 215, 200 y $190{ }^{\circ} \mathrm{C}$ respectivamente, con una velocidad de $40 \mathrm{rpm}$.

Etapa 5: Elaboración de probetas para realizar las pruebas de resistencia al impacto y dureza del material compuesto.

La elaboración de las placas para cortar y maquinar las probetas para la resistencia al impacto, tensión y dureza se realizaron en una prensa de platos calientes marca Hull modelo 70-AX-2299, mostrada en la figura 6.

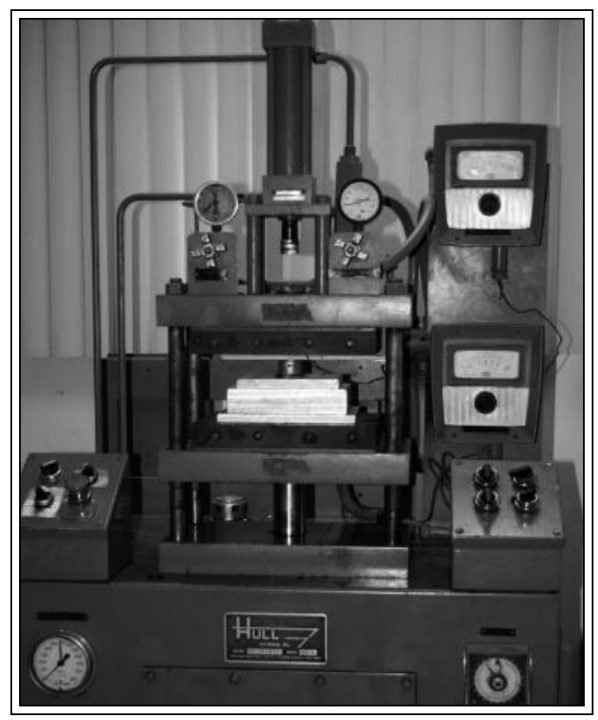

Figura 6.

Máquina de moldeo por transferencia de platos calientes, marca Hull. 
En esta etapa se moldeó por transferencia placas de $6.35 \mathrm{~mm}$ de espesor en la prensa de platos calientes a una presión de transferencia de $8200 \mathrm{KPa}$ y con una temperatura en los platos de $200^{\circ} \mathrm{C}$, de donde se maquinaron las probetas para realizar las pruebas de dureza shore D según la norma ASTM D2240 y de resistencia al impacto según la norma ASTM D256-93.

Para la realización de las probetas se utilizó la norma ASTM D4703d que es la indicada en la preparación de probetas por moldeo en prensa. En el proceso se observó que fue necesario el control preciso de las variables: temperatura de las tres zonas del extrusor, temperatura y presión del moldeo por transferencia.

Para la medición de resistencia al impacto se realizaron 10 probetas para cada combinación de factores, las cuales tienen la forma y dimensiones mostradas en la figura 7 y en la figura 8 las probetas elaboradas. Las probetas obtenidas tuvieron un acondicionamiento por 24 horas a $23 \mathrm{oC}$ con una humedad relativa del $50 \%$.
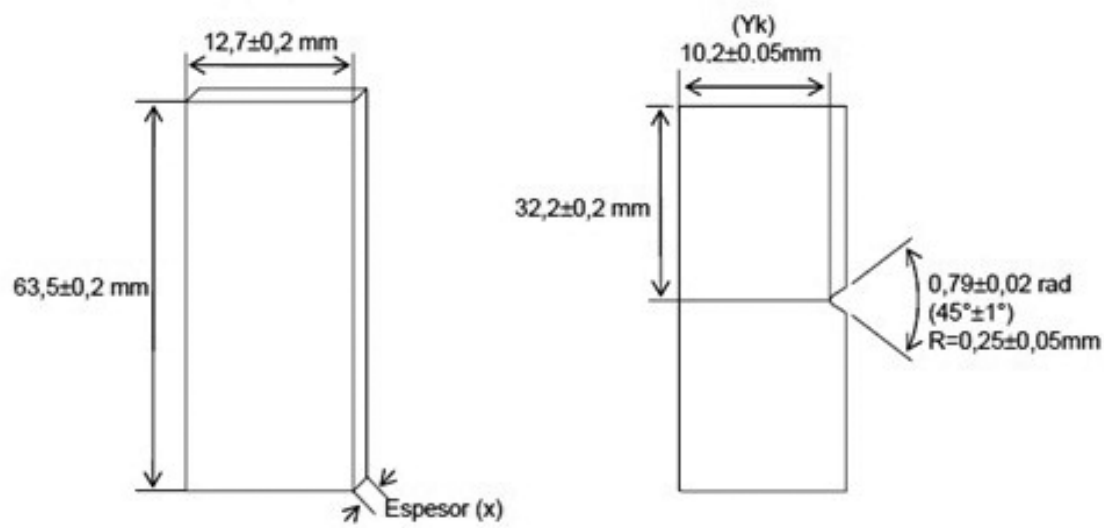

Figura 7. Probeta para pruebas de impacto, según la Norma ASTM D256-93.

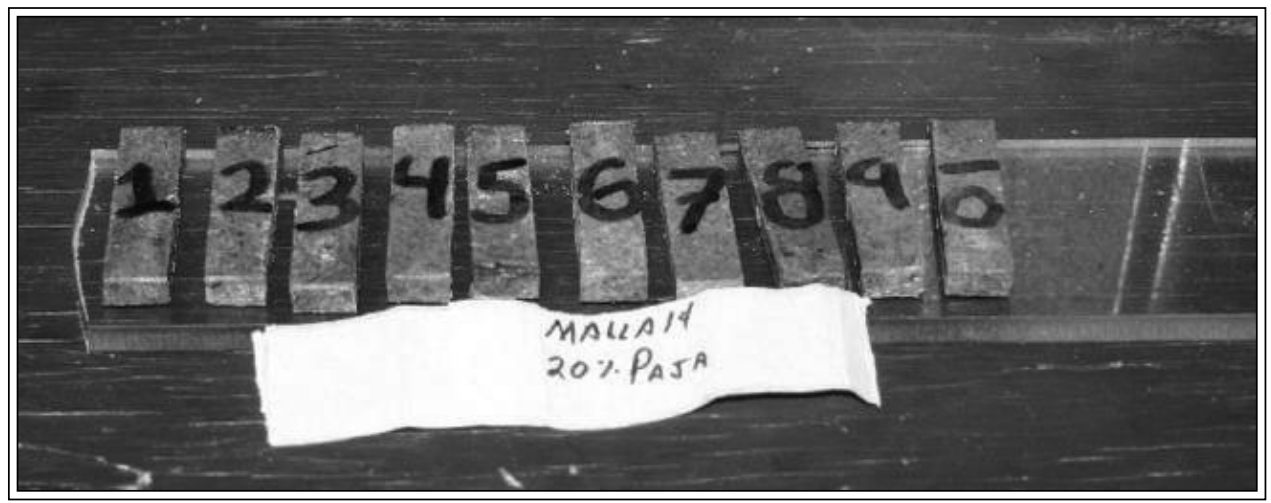

Figura 8. Probetas para las pruebas de impacto. 
A cada probeta se le hizo una muesca con una fresadora transversal cuidando que no hubiese calentamiento. Posteriormente, se utilizó una máquina de impacto Monsanto Tensómetro de doble brazo, mostrado en la figura 9, que utiliza una velocidad de impacto de $3.5 \mathrm{~m} / \mathrm{s}$ en la prueba Charpy, para medir la energía absorbida en la ruptura de las muestras, el valor de esta energía es definido como la diferencia entre la energía inicial del péndulo y la energía remanente en el péndulo después de romper la muestra.

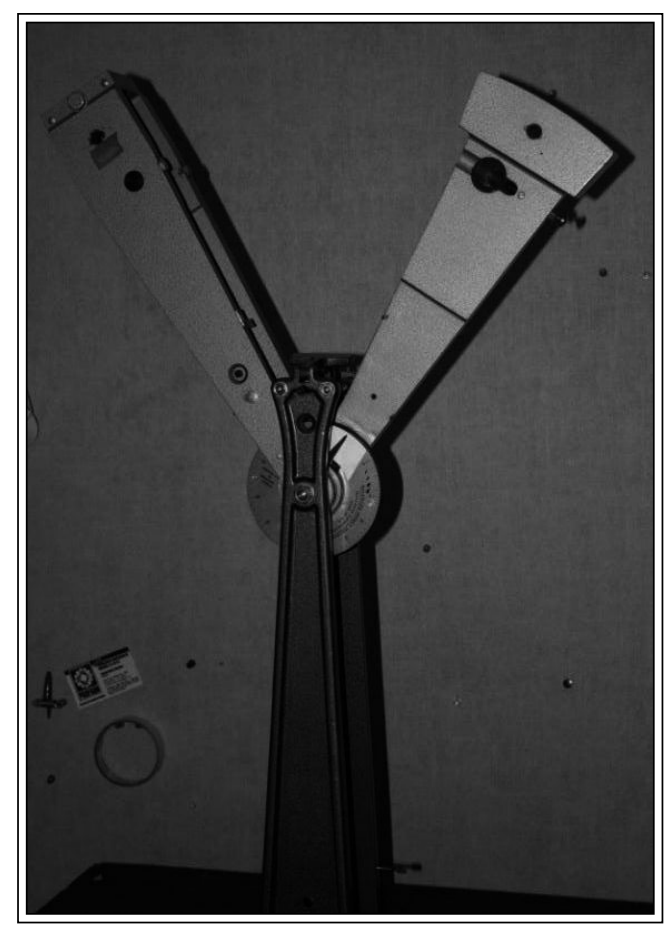

Figura 9. Máquina para realizar las pruebas de impacto.

La resistencia al impacto se determinó por la relación

$$
\mathrm{E}=\frac{\mathrm{A}_{\mathrm{k}} \times 10^{3}}{\mathrm{x} \cdot \mathrm{Y}_{\mathrm{k}}}
$$

Dónde:

EI: Energía de impacto.

Ak: Energía de impacto absorbida por el espécimen, en Joules.

x: Espesor de la muestra.

Yk: Distancia entre el fondo de la ranura y la cara opuesta a ésta en mm. 
Se midió dureza Shore D en las probetas obtenidas para las diferentes combinaciones de paja y tamaño de partícula. Esta prueba se realizó según la norma ASTM D2240. Se utilizó un durómetro marca SUNDOO modelo LX-D para plásticos duros.

Las dimensiones de las probetas utilizadas para la medición de dureza fueron de un espesor de $6.7 \mathrm{~mm}$ con superficies planas. Estas probetas se acondicionaron durante 3 horas a $23{ }^{\circ} \mathrm{C}$ con una humedad del $50 \%$. En la Figura 10 se muestran las probetas para las pruebas de dureza.

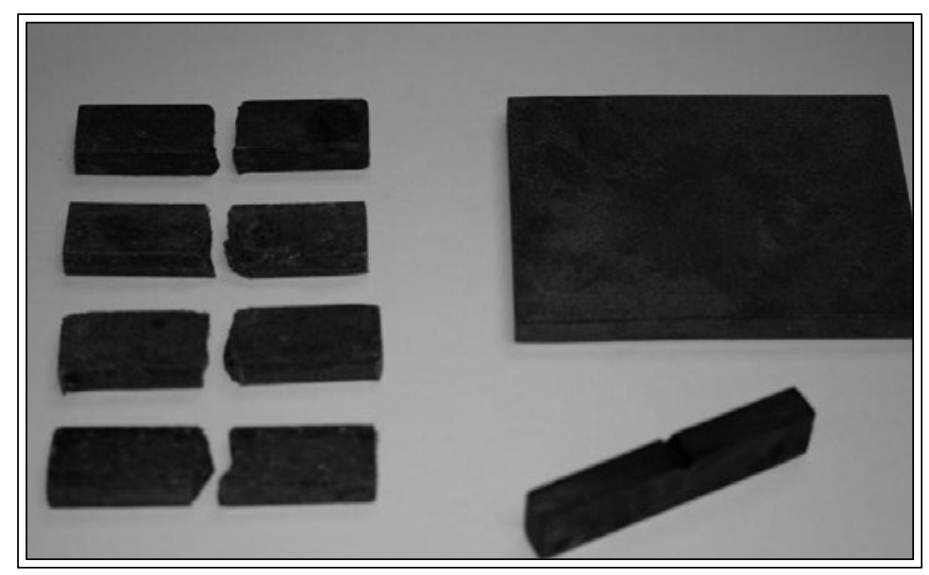

Figura 10. Probetas para las pruebas de dureza.

Se muestran en la Figura 11 placas de madera plástica con PET y $30 \%$ de paja con malla 14, 30 y 40 respectivamente y en la Figura 12 placas elaboradas solo con PET de post consumo.

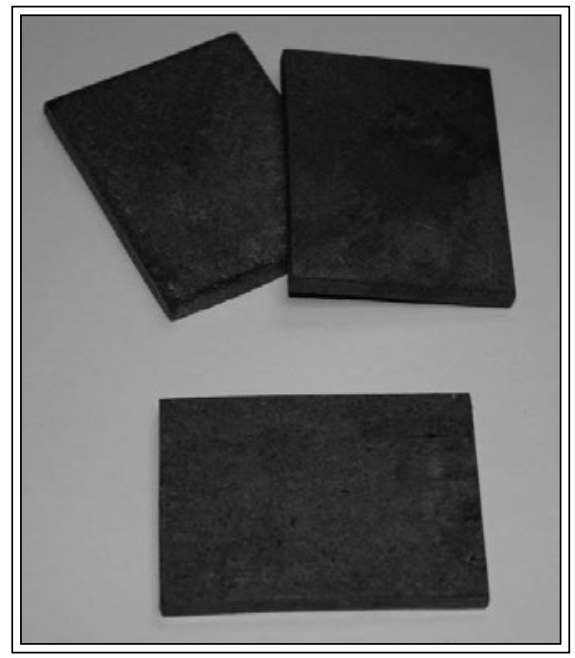

Figura 11. Placas de madera plástica con 30\% de paja (malla 14, 30 y 40) y PET 


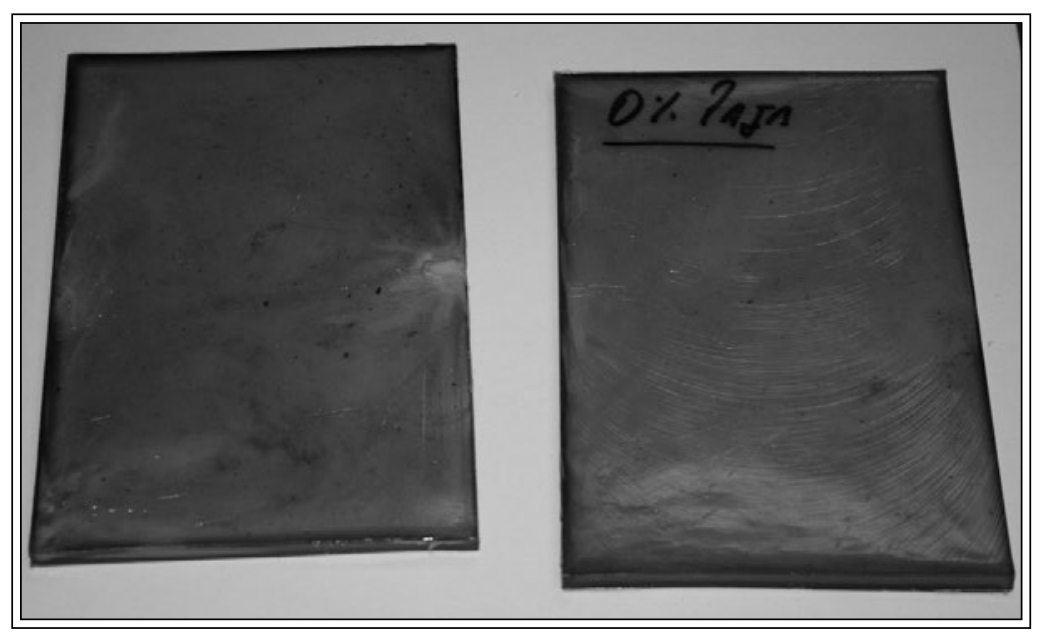

Figura 12. Placas elaboradas con PET de post consumo.

\section{Resultados y discusión}

En la Tabla 2 se muestran los valores promedio de la resistencia al impacto en las probetas obtenidas y la desviación estándar en cada una de las combinaciones realizadas. Es posible observar que para los diferentes tamaños de malla, al incrementar el porcentaje de paja se obtiene mayor resistencia al impacto. Sin embargo, con $40 \%$ de paja la mezcla muestra muchos problemas de procesabilidad y no fue posible realizar esta combinación.

De las probetas fracturadas, se observó que para todos los tamaños de malla las probetas presentaron fracturas demasiado frágiles. En algunos casos las probetas se rompieron en más de dos pedazos.

\begin{tabular}{|c|c|c|c|c|c|c|c|c|}
\hline / & \multicolumn{7}{|c|}{$\begin{array}{c}\text { Energía de Impacto absorbida Promedio (KJ/m2) } \\
\text { Tamaño de partícula, Malla }\end{array}$} \\
\hline $\begin{array}{c}\% \text { de } \\
\text { paja }\end{array}$ & 14 & DE & 20 & DE & 30 & DE & 40 & DE \\
\hline 10 & 52.489 & 12.674 & 45.138 & 9.627 & 49.591 & 7.541 & 44.455 & $\begin{array}{c}9.182 \\
20\end{array}$ \\
\hline 20 & 53.482 & 12.009 & 44.054 & 8.768 & 51.563 & 14.484 & 63.189 & 15.359 \\
\hline 30 & 62.079 & 8.810 & 46.916 & 12.669 & 59.924 & 17.899 & 56.617 & 13.660 \\
\hline
\end{tabular}

DE = Desviación estándar 
Se observó lo siguiente:

1. Al incrementar el porcentaje de paja se obtiene:

- Mejora en la resistencia al impacto.

- Se dificulta el mezclado en el extrusor.

- Aumentan la cantidad de vapores emitidos durante mezclado y moldeado.

- Se quema con mayor facilidad la paja.

2. No se realizaron las pruebas con el $40 \%$ de paja de trigo porque la placa obtenida presentaba una consistencia muy baja.

La energía de impacto absorbida por la madera está en el intervalo de $36-250 \mathrm{~kJ} / \mathrm{m} 2$.

Adicionalmente se realizaron combinaciones de polipropileno (PP) y polietileno de alta densidad (HDPE) con PET y paja de trigo, los valores de la resistencia al impacto en las probetas obtenidas se muestran en la Tabla 3.

La incorporación de Polipropileno y Polietileno de alta densidad en la mezcla de paja de trigo y PET en las pruebas de impacto permitieron incrementar la energía de fractura y reducir la fragilidad. La incorporación de estos plásticos a la mezcla facilita considerablemente la procesabilidad en el mezclado y capacidad de moldeo.

Tabla 3. Valores promedio de la resistencia al impacto en las probetas obtenidas de la mezcla de PET y paja con otros componentes.

\begin{tabular}{|c|c|c|}
\hline \multirow{2}{*}{ Probetas } & \multicolumn{2}{|c|}{$\begin{array}{c}\text { Energía de Impacto absorbida Promedio (KJ/mm2) } \\
\text { Tamaño de partícula, malla }\end{array}$} \\
\cline { 2 - 3 } & 40 & Desviación estándar \\
\hline PET & 43.285 & 8.879 \\
\hline PET + 20\% de polipropileno & 59.394 & 3.502 \\
\hline PET + Paja + polietileno de alta densidad & 66.413 & 4.077 \\
\hline
\end{tabular}

La incorporación de polipropileno (PP) y polietileno de alta densidad (HDPE) en la mezcla:

- Incrementa la energía de fractura.

- Reduce la fragilidad.

- Facilita la procesabilidad en el mezclado.

- Reduce la cantidad de humos emitidos

- Mejora la capacidad de moldeo.

- Reducen porcentajes de cristalización del PET 
En la Tabla 4 se observa en los tamaños de partícula con malla 20, 30 y 40 que al incrementar el porcentaje de paja la dureza decae, sin embargo, con el tamaño de partícula de malla 14 no se aprecia este comportamiento, por lo que, se considera que para este tamaño de partícula no es posible lograr buena unión entre la paja y el PET, ya que este no recubre completamente la partícula de paja.

Tabla 4. Valor de dureza en las probetas obtenidas.

\begin{tabular}{|c|c|c|c|}
\hline$/$ & \multicolumn{3}{|c|}{ Valor de dureza } \\
Tamaño de partícula, Malla \\
\hline$\%$ DE PAJA & 14 & 20 & 30 \\
\hline 10 & 53 & 57 & 56 \\
\hline 20 & 68 & 62 & 59 \\
\hline 30 & 66 & 66 & 62 \\
\hline 40 & 68 & 65 & 60 \\
\hline
\end{tabular}

Se muestran en la Tabla 5 los valores de dureza de las probetas realizadas solo con PET y puede observarse que son las que presentan mayor dureza, cabe mencionar que la dureza del PET virgen es 63 Shore D, menor que la dureza obtenida en este trabajo para el PET reciclado, debido a las pérdidas de las propiedades y reducción de viscosidad intrínseca del reciclado mismo. La dureza de las probetas con polietileno de alta densidad y polipropileno no son las más altas, pero es importante resaltar la buena miscibilidad alcanzada entre la paja de trigo, el PET y el polipropileno o en su cado el Polietileno de alta densidad (HDPE).

Tabla 5. Valor de dureza en las probetas obtenidas de la mezcla de PET y paja con otros componentes. Tamaño de partícula, Malla 40.

\begin{tabular}{|c|c|}
\hline Probetas & Valor de dureza \\
\hline PET & 78 \\
\hline PET $+10 \%$ HDPE $+10 \%$ Paja & 64 \\
\hline PET $+10 \%$ PP $+10 \%$ Paja & 66 \\
\hline
\end{tabular}

Las probetas realizadas con PET presentan mayor dureza, la dureza del PET virgen es 63 Shore D, menor que la dureza obtenida en este trabajo para el PET reciclado, debido a las pérdidas de las propiedades y reducción de viscosidad intrínseca del reciclado mismo. 


\section{Conclusiones}

Se logró el objetivo general de la investigación al desarrollar un material compuesto de polietileno tereftalato (PET) de post consumo y paja de trigo, para la elaboración de madera plástica.

Se obtuvo una placa de material compuesto con refuerzo de paja de trigo y PET de post consumo como una alternativa para el consumidor de la región de madera en el sector construcción de viviendas y muebles. Así como una alternativa de uso de la paja de trigo para el agricultor del valle de Mexicali y para el PET y otra opción más para la reducción de la tala de árboles, al elaborar madera plástica con dos materiales de desecho.

El material compuesto obtenido, no presenta poros, se puede: taladrar, lijar, pulir, cortar con sierra manual o eléctrica y moldear por extrusión y por transferencia.

Debido a que la paja y el PET no son completamente miscibles se mantienen dos fases en la mezcla cuyo tamaño dependerá de las concentraciones y tamaños de partícula generando con esto variaciones en las resistencias al impacto y dureza del compuesto.

Al fundir el PET reciclado la viscosidad intrínseca disminuye, sus moléculas se rompen reduciéndose el peso molecular y con ello se incrementa la fragilidad y se reduce la flexibilidad del PET reciclado, la incorporación de paja de trigo con diferentes tamaños de malla no le regresa las propiedades de flexibilidad al PET pero le ayuda a incrementar su resistencia al impacto.

Se considera que la incorporación de polipropileno o polietileno de alta densidad mejoran considerablemente la procesabilidad de la mezcla de PET y paja, así como su capacidad de moldeo, por lo cual se propone como resultado de este proyecto el uso de mezclas de PET reciclado y paja de trigo con polipropileno o polietileno de alta densidad.

Los resultados obtenidos permiten establecer procesos de mezclado y moldeo que logren producciones a mayor escala y así ofrecer una opción viable para evitar la quema de la paja de trigo y reducir la contaminación por botellas de PET. La madera plástica obtenida puede utilizarse para la fabricación de muebles, andamios, molduras y fabricación de ladrillos de ensamble para construcción rápida

\section{Comentarios}

La problemática del rehúso de materiales de desecho como el PET y la paja de trigo, es una realidad en la región y representa una necesidad y una área de oportunidad para los docentes y estudiantes del Instituto Tecnológico de Mexicali en Baja California, México, por lo que, además de estar conscientes de ella, debemos 
contribuir para su disminución, razón por la cual en este proyecto se procesó el PET y la paja de trigo con el fin de darles otro uso y reducir la presencia de ambos contaminantes en el medio ambiente.

\section{Recomendaciones}

Es importante mencionar, que en esta investigación se determinó la formulación óptima entre PET y paja de trigo, así como el proceso para la elaboración de un producto. Observando el acabado y consistencia del producto obtenido se considera que podría sustituir a la madera de aserrín comprimido en muchas de sus aplicaciones en la construcción de muebles, sin embargo, es necesaria una caracterización mecánica del producto con la realización de pruebas: de resistencia a la tensión, a la compresión y a la flexión, así como de absorción de humedad. Además habría que realizar pruebas de procesabilidad en la fabricación de muebles como: clavado, machuelado (formado de cuerdas), corte con sierra, adhesión con colas, lijado y pulido, que pudieran junto con la caracterización mecánica establecer argumentos comparativos del producto obtenido con la madera de aserrín comprimido y así tomar decisiones para el diseño en carpintería y en otras aplicaciones.

\section{Agradecimientos}

A la Secretaría de Educación Pública por haber proporcionado el recurso financiero al Cuerpo Académico de Educación Superior en Ingeniería del Instituto Tecnológico de Mexicali, Baja California, México, para realizar el Proyecto en el marco de la Convocatoria de Fortalecimiento a los Cuerpos Académicos 2010.

Al Dr. Benjamín Valdez Salas, Director del Instituto de Ingeniería de la Universidad Autónoma de Baja California, México, por su apoyo en la realización de algunas pruebas en la madera plástica. 


\section{Referencias}

Chon, C. T. (1980). Complex moduli of aligned discontinuos fibre-reinforced polymer compositives. Journal of Materials, 15.

Cox, H. (Marzo de 1952). The elasticity and strength of paper and other fibrous materials. Recuperado el 8 de abril de 2011, de British journal of applied physics: http://iopscience.iop.org/0508-3443/3/3/302/pdf/0508-3443_3_3_302.pdf.

Flores, L. (2010). Oportunidades de Innovación con Materiales Reforzados. Tecnología del Plástico, Vol. 25, Edición 3.

García, C. (2010). Potencial y Retos de los Compuestos Plásticos y Fibras Naturales. Tecnología del Plástico, Vol. 25, Edición 3, 23.

García, S. (Abril de 2006). Productos PET, una Oprtunidad de Negocio. Reforma, pág. 6 .

Plueddemann, E. (1974). Mechanism of adhesion through silane coupling agents. New York: Academic Press.

Quintero, N. M. (2008). Contaminación y Control de las Quemas Agrícolas en Imperial, California y Mexicali. Región y Sociedad, Vol. XX, 4-24.

Sato, Y. F. (Septiembre de 1962). A Molecular Theory of Filler Reinforcement Based on the Concept of Internal Deformation. Recuperado el 7 de abril de 2011, de Rubber Chemistry and Technology, vol. 35 (4), pp 865-876: http:// rubberchemtechnol.org/doi/pdf/10.5254/1.3539978 